ARTICLE OPEN

\title{
Quantum simulations with multiphoton Fock states
}

\author{
T. J. Sturges (ID) ${ }^{1}$, T. McDermott ${ }^{1}$, A. Buraczewski ${ }^{1}{ }^{1}$, W. R. Clements ${ }^{2}$, J. J. Renema ${ }^{3}$, S. W. Nam ${ }^{4}$, T. Gerrits ${ }^{4}$, A. Lita ${ }^{4}$, W. S. Kolthammer ${ }^{2}$,
} A. Eckstein ${ }^{2}$ I. A. Walmsley ${ }^{2}$ and M. Stobińska (D)

Quantum simulations are becoming an essential tool for studying complex phenomena, e.g. quantum topology, quantum information transfer and relativistic wave equations, beyond the limitations of analytical computations and experimental observations. To date, the primary resources used in proof-of-principle experiments are collections of qubits, coherent states or multiple single-particle Fock states. Here we show a quantum simulation performed using genuine higher-order Fock states, with two or more indistinguishable particles occupying the same bosonic mode. This was implemented by interfering pairs of Fock states with up to five photons on an interferometer, and measuring the output states with photon-number-resolving detectors. Already this resource-efficient demonstration reveals topological matter, simulates non-linear systems and elucidates a perfect quantum transfer mechanism which can be used to transport Majorana fermions.

npj Quantum Information (2021)7:91 ; https://doi.org/10.1038/s41534-021-00427-w

\section{INTRODUCTION}

Quantum simulations boost the development of topological materials ${ }^{1}$, quantum transport ${ }^{2}$ and quantum algorithms ${ }^{3}$ for the benefit of low-power electronics ${ }^{4}$, spintronics ${ }^{5}$ and quantum computing ${ }^{6}$. They employ intricate quantum interference of light or matter particles. This is a challenging task: the difficulty arises from the fundamental constraint that all interfering quanta must be indistinguishable ${ }^{7}$. Violating this demand precludes the observation of such coherent phenomena in larger scales, in terms of particle number and duration.

So far, protocols have mainly relied on the use of three distinct quantum states: numerous qubits implemented by superconducting circuits $^{8}$ and electronic states of trapped ions ${ }^{9,10}$; coherent states of photons ${ }^{11}$ and atoms (Bose-Einstein condensates) ${ }^{12}$; and multiple single-particle Fock (number) states distributed among many modes in photonic waveguides ${ }^{13-18}$ and optical lattices ${ }^{19}$. Thus, simulations have never seriously profited from interference of multi-particle Fock states, even though the importance of this regime has been recognised ${ }^{20}$, and the first attempt to mimic it with many-body systems was made ${ }^{21}$.

Here we experimentally and theoretically demonstrate that multiphoton Fock state interference can be useful for quantum simulations that address applications of high impact. We employ a single multiport interferometer with a tunable parameter that encodes time to reach a late state of evolution. This approach allows us to avoid error accumulation that is typical for methods that build on numerous quantum gates or steps of a quantum algorithm ${ }^{22-27}$. This is a counterpart of a hardware-encoded operation in classical computation, where a single binary instruction realizes a complex algorithm consisting of several primitive steps in one tick of a system clock. Remarkably, utilisation of photon-number detection in our setup grants us access to a hidden non-linearity induced by a projective measurement, a technique that enables universal quantum computation with linear optics ${ }^{28,29}$.

As a concrete example, we consider a quantum gate architecture that consists of a single beam splitter; however, this approach can be generalised to more complex interferometric networks. Our idea, shown in Fig. 1a-c, is based on overlapping two multiphoton Fock states, $|I\rangle_{a}$ and $|S-I\rangle_{b}$ (I photons in mode $a$ and $S-l$ in mode $b$ ), on a beam splitter with tunable reflectivity $r$ which programmes the simulation duration. We then collect photon statistics at its outputs.

The scope of our example embraces a chain of $S+1$ twolevel spins that initially contains just one spin excited, and that is subjected to an $\mathrm{XY}$ interaction. The excitation probabilities at its sites after the interaction duration are determined by the output photon statistics. These mappings are based on a solid mathematical grounding known as the Schwinger representation which links quantum harmonic oscillators with representations of spin Lie algebra su(2). See Supplementary Note 1 for details.

Our platform also allows us to simulate certain classes of fermionic systems, e.g. a non-linear Su-Schrieffer-Heeger (SSH) model $^{30}$, obtained from the XY spin chain by a Jordan-Wigner transformation $^{31}$. Furthermore, we can map to Bogoliubov-de Gennes Hamiltonians, simulating many-body systems beyond the single-excitation subspace, e.g. a p-wave superconducting chain (Kitaev model) ${ }^{32}$, and the transverse-field Ising model ${ }^{9}$.

Due to recent advances in photon-number-resolved detection, we were able to employ transition-edge sensors (TESs) ${ }^{33}$ to count photons exiting the beam splitter. Amazingly, TES measurements correspond to single-site-resolved detection in the chain. The use of TESs is crucial, as Fock state quantum interference is evidenced by photon bunching. For example, two identical photons impinging on a balanced beam splitter leave in a superposition of two-photon Fock states, with both always being detected in the same output port. This is known as the Hong-Ou-Mandel (HOM) effect ${ }^{34}$ whose generalised form can be observed for higher-order Fock states if they are prepared in similar polarisation, spectral and spatio-temporal modes ${ }^{35}$, as shown in Fig. $1 \mathrm{~d}$.

\footnotetext{
${ }^{1}$ Institute of Theoretical Physics, University of Warsaw, Warsaw, Poland. ${ }^{2}$ Clarendon Laboratory, University of Oxford, Oxford, UK. ${ }^{3}$ Complex Photonic Systems (COPS), MESA+ Institute for Nanotechnology, University of Twente, Enschede, Netherlands. ${ }^{4}$ National Institute of Standards and Technology, Boulder, CO, USA. ${ }^{2}$ email: magdalena. stobinska@gmail.com
} 


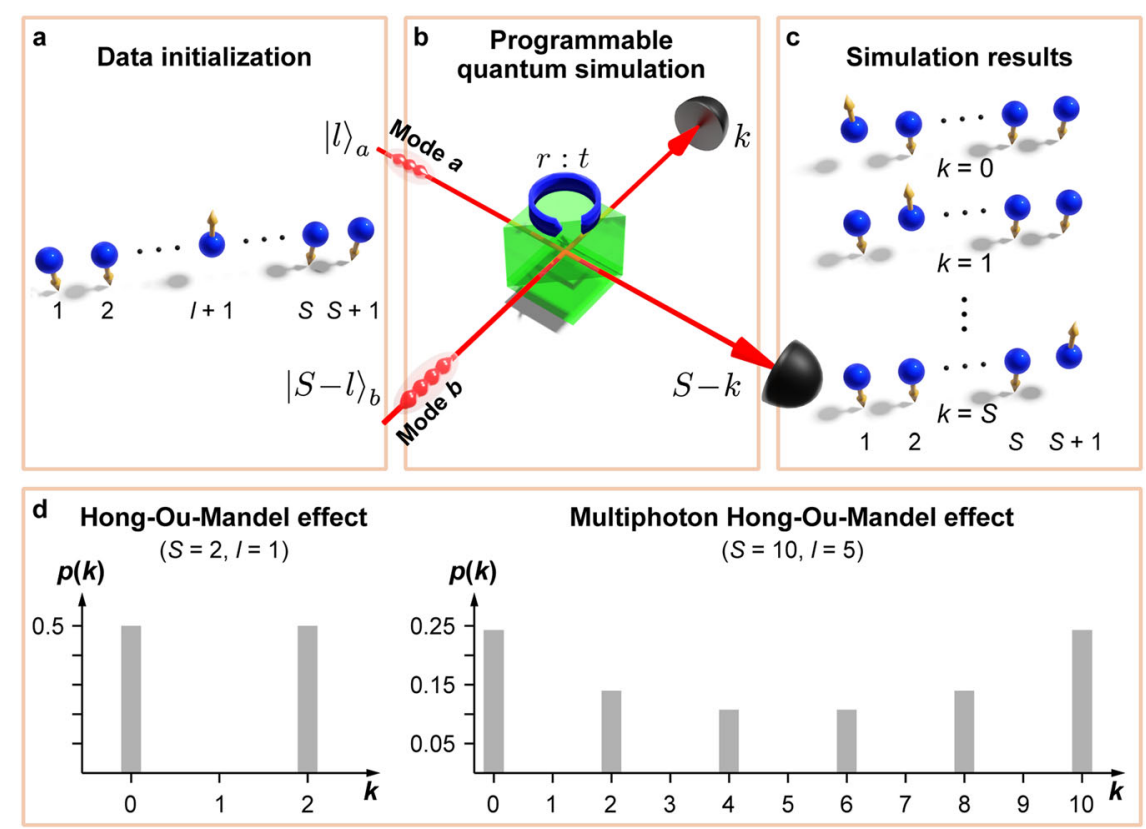

Fig. 1 Photonic quantum simulations with Fock state interference. a Fock states $|I\rangle_{a}|S-I\rangle_{b}$ encode the $(I+1)$ th spin excitation in a $\frac{1}{2}$-spin chain with $S+1$ sites. b A beam splitter with reflectivity $r$ models an XY-type of interaction in the chain that lasts 2 arcsin $\sqrt{r}$. c The likelihood of detecting $k$ and $S-k$ photons in its output ports $p(k)$, simulates the excitation probability of the $k$ th spin in the chain as a result of the interaction. d The statistics $p(k)$ originates from fundamental indistinguishability of several scenarios that occur to interfering Fock states which classically are exclusive but quantum-mechanically are coexisting, and amount to the same partitioning of incoming photons into two exit ports. Events for which quantum probability amplitudes add up non-destructively are registered more often than others.

\section{RESULTS}

\section{Fock state quantum simulations}

The Fock state quantum simulations build on a beam-splitter interaction $U_{\mathrm{BS}}^{(r)}=e^{-i \theta(r) H_{\mathrm{BS}}}$, guided by the Hamiltonian

$H_{\mathrm{BS}}=\frac{1}{2}\left(a^{\dagger} b+a b^{\dagger}\right)$,

where $a^{\dagger}$ and $b^{\dagger}$ denote photonic creation operators that act on the interferometer input modes. The reflectivity $r$, defined as the probability of reflection of a single photon, encodes the interaction time $\theta(r)=2 \arcsin \sqrt{r}$. For entries $|I\rangle_{a}$ and $|S-I\rangle_{b}$, the computational output from the beam splitter and detectors is ${ }^{36}$

$p(k)=\left|\left\langle k, S-k\left|U_{\mathrm{BS}}^{(r)}\right| I, S-I\right\rangle\right|^{2}=\left|\phi_{k}^{(r)}(I-S r, S)\right|^{2}$,

where $\phi_{k}^{(r)}(x, S)$ is the Kravchuk function ${ }^{37}$.

We selected three distinct examples of simulations, shown in Fig. 2, for experimental demonstration. The first $\mathbf{a}$, uses input data initialised to $\left|\frac{S}{2}\right\rangle_{a}\left|\frac{S}{2}\right\rangle_{b}$ and the setting of $r=0.5$. For the second and third $\mathbf{b} \& \mathbf{c}$, we set $|0\rangle_{a}|S\rangle_{b}$ and repeated the computation several times while gradually increasing $r$. While for the second programme one can use any value of $S$, the third one runs exclusively for an odd number of photons.

\section{Edge states in non-linear systems}

Interpretation of the outcomes of our quantum programmes becomes straightforward if we consider matrix representations of $H_{\mathrm{BS}}$ and of the Hamiltonian describing a general chiral XY $\frac{1}{2}$-spin chain $H_{\mathrm{XY}}=\sum_{n=1}^{S+1} \frac{J_{n}}{2}\left(\sigma_{n}^{x} \sigma_{n+1}^{x}+\sigma_{n}^{y} \sigma_{n+1}^{y}\right)$, where $\sigma_{n}^{x}$ and $\sigma_{n}^{y}$ are the Pauli operators acting on the $n$th spin. In the single excitation subspace spanned by the states $|n\rangle=\sigma_{n}^{+}\left|\downarrow_{1}, \ldots, \downarrow_{s+1}\right\rangle$, where $\sigma_{n}^{+}=(1 / 2)\left(\sigma_{n}^{x}+i \sigma_{n}^{y}\right)$ is the raising operator, the latter has matrix elements $\left[\mathbf{H}_{\mathrm{XY}}\right]_{m n}^{\text {Spin }}=$ $\left\langle m\left|H_{\mathrm{XY}}\right| n\right\rangle=J_{n-1} \delta_{n, m+1}+J_{m-1} \delta_{m, n+1}$, where $\delta_{i, j}$ denotes the Kronecker delta. The elements of $H_{\mathrm{BS}}$ in the Fock state basis are given by $\left[\mathbf{H}_{\mathrm{BS}}\right]_{n m}^{\text {Fock }}=\left\langle n, S-n\left|H_{\mathrm{BS}}\right| m, S-m\right\rangle$. The two representations are identical, $\left[\mathbf{H}_{\mathrm{BS}}\right]_{n m}^{\text {Fock }}=\left[\mathbf{H}_{\mathrm{XY}}\right]_{n m}^{\text {Spin }}$, when we set the spin couplings to $J_{n}=\frac{1}{2} \sqrt{n(S+1-n)}$. As these amplitudes are non-periodic, this chain lacks translational invariance. This precludes the usual Fourier space methods used for characterising topological insulators. Remarkably, photon statistics measured behind the beam splitter is capable of simulating this noncrystalline system. The existence of topologically non-trivial states is indicated here by the fact that the Hamiltonian belongs to the chiral orthogonal (BDI) class of Altland-Zirnbauer symmetry classes, characterised by a $\mathbb{Z}$ topological invariant. Our first programme performs a real-space study of this system and computes probabilities that describe its zero-energy eigenmode, $\left|\Psi_{0}\right\rangle=\sum_{k=0}^{S} e^{-i \frac{\pi}{2}(S / 2-k)} \phi_{k}^{(1 / 2)}(0, S) \sigma_{k+1}^{+}\left|\downarrow_{1}, \ldots, \downarrow_{S+1}\right\rangle$. Unlike the typical edge states which are exponentially peaked at the ends of a quantum wire, these two edge states are weakly localised and plateau to a constant value in the bulk, given by $\frac{4}{\pi S \sqrt{1-(2 k / S-1)^{2}}}$, as outlined in Fig. 2a. The intensity-dependent amplitudes $J_{n}$ render $H_{\mathrm{XY}}$ a generalisation of the seminal Su-Schrieffer-Heeger (SSH) model $^{30}$ to the non-linear regime ${ }^{38}$. See Supplementary Notes 2 and 3 for details.

\section{Perfect state transfer}

The $X Y$ spin chain with these couplings has been extensively studied in the literature due to its remarkable property of facilitating the perfect transfer of an arbitrary quantum state ${ }^{16,17,39,40}$. Our quantum simulation provides an insight into this system from which the perfect transfer becomes self-evident. The equivalence of $H_{\mathrm{BS}}$ and $H_{\mathrm{XY}}$ matrix representations implies the correspondence between interactions generated by these Hamiltonians, $U_{\mathrm{BS}}^{(r)}$ and $U_{\mathrm{XY}}(t)=e^{-i t H_{\mathrm{XY}}}$, respectively. Mathematically, the beam-splitter interaction in the Fock state basis amounts to an $a$-fractional Quantum Kravchuk-Fourier transform ( $a$-QKT) of the input state with fractionality $a=\frac{4}{\pi} \arcsin \sqrt{r}^{36}$. As 2-QKT is the 

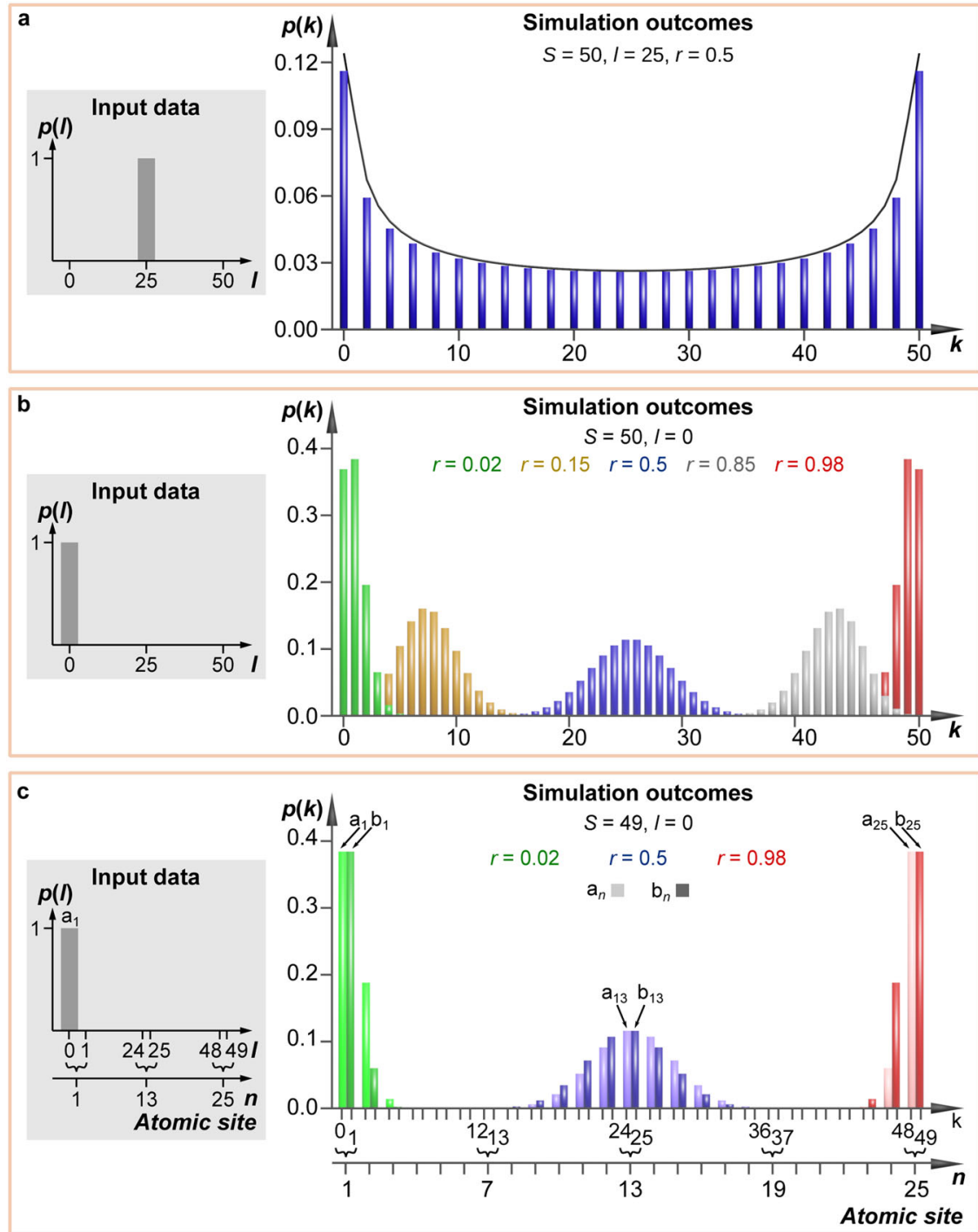

Fig. 2 Encoding the outcomes of quantum simulations in photon statistics. We repeatedly overlap two Fock states on a beam splitter of reflectivity $r$ to collect the photon statistics in its exit ports, $p(k)$. They directly provide the results of computation carried out by quantum interference. a The first programme uses $\left|\frac{S}{2}\right\rangle_{a}\left|\frac{S}{2}\right\rangle_{b}$ and $r=0.5$, revealing weakly localised edge states with a non-decaying envelope (black line), which closely resemble topological states in a non-linear SSH model. b The second programme is run for $|0\rangle_{a}|S\rangle_{b}$ and several values of $r$, demonstrating that perfect quantum wave packet transfer in a linear register results from mirror reflection of the input state w.r.t. the register centre. c For an odd $S$, this programme additionally simulates the perfect transfer of Majorana fermions $a_{n}$ and $b_{n}$ in a p-wave superconductor over $\frac{S+1}{2}$ atomic sites. The bars located at even $k$ (light green, blue, and red) correspond to the mode $a_{n}$ with $n=k / 2+1$, while those located at odd $k$ (dark green, blue, and red) to the mode $b_{n}$ with $n=(k+1) / 2$.

spatial inversion operator ${ }^{37}$, so is $U_{\mathrm{XY}}(t)$ at $t=\pi$. Therefore, the transfer is an effect of mirror reflection of a quantum state w.r.t. the chain centre. Proving this fact was tricky within the framework of spin chains, whereas it is an evident conclusion from our photonic simulations. We note that $a=2$ implies interference on a perfectly reflecting beam splitter $(r=1)$ which swaps input states at its outputs. To demonstrate this behaviour, in our second programme, we simulated the state transfer of a strongly localised edge state, typical of e.g. the SSH model. The initial Fock state $|0\rangle_{a}|S\rangle_{b}$ is gradually transformed to $|S\rangle_{a}|0\rangle_{b}$ for increasing $r$, as shown in Fig. 2b. Derivations are presented in Supplementary Note 4.

\section{Generalised Majorana modes}

Multiphoton Fock state interference also facilitates the simulation of many-body systems that are not restricted to a single excitation subspace. For example, a $\mathrm{p}$-wave superconducting chain (Kitaev model) ${ }^{32}$ is described by the mean field Hamiltonian
$H_{\mathrm{K}}=\sum_{n=1}^{N}\left\{-\mu_{n}\left(c_{n}^{\dagger} c_{n}-1 / 2\right)-t_{n}\left(c_{n+1}^{\dagger} c_{n}+c_{n}^{\dagger} c_{n+1}\right)+\Delta_{n}\left(c_{n+1}^{\dagger} c_{n}^{\dagger}+\right.\right.$ $\left.\left.c_{n} c_{n+1}\right)\right\}$, where $c_{n}^{\dagger}$ and $c_{n}$ are creation and annihilation operators for electrons on the $n$th atomic site, while $\mu_{n}, t_{n}$ and $\Delta_{n}$ are site dependent chemical potentials, hopping amplitudes and superconducting pairing potentials, respectively. This Hamiltonian may be expressed in the form $H_{\mathrm{K}}=\frac{1}{2} X^{\dagger} \mathbf{H}_{\mathrm{BdG}} X$ where $X=$ $\frac{1}{\sqrt{2}}\left(a_{1},-i b_{1}, a_{2},-i b_{2}, \ldots a_{N},-i b_{N}\right)^{\top}$ is a Nambu spinor and $\mathbf{H}_{\mathrm{BdG}}$ is the Bogoliubov-de Gennes Hamiltonian matrix, in the basis of Majorana operators $a_{n}=c_{n}+c_{n}^{\dagger}$ and $b_{n}=i\left(c_{n}^{\dagger}-c_{n}\right)$. The beamsplitter Hamiltonian in the Fock state basis $\mathbf{H}_{\mathrm{BS}}$ is identical to $\mathbf{H}_{\mathrm{BdG}}$ for the parameters $\mu_{n}=J_{2 n-1}, t_{n}=\Delta_{n}=\frac{J_{2 n}}{2}$, where $2 N=S+1$. This correspondence allows one to simulate the Heisenberg evolution of the Majorana operators over the interaction time $\theta(r)$, as well as the evolution of the real fermion operators $c_{n}$ and $c_{n}^{\dagger}$, by using linear superpositions of Fock states as input. In particular, the evolution of the operators $a_{n}$ and $-i b_{n}$ is encoded by the evolution of the photonic modes $|2(n-1)\rangle_{a}|S-2(n-1)\rangle_{b}$ and 


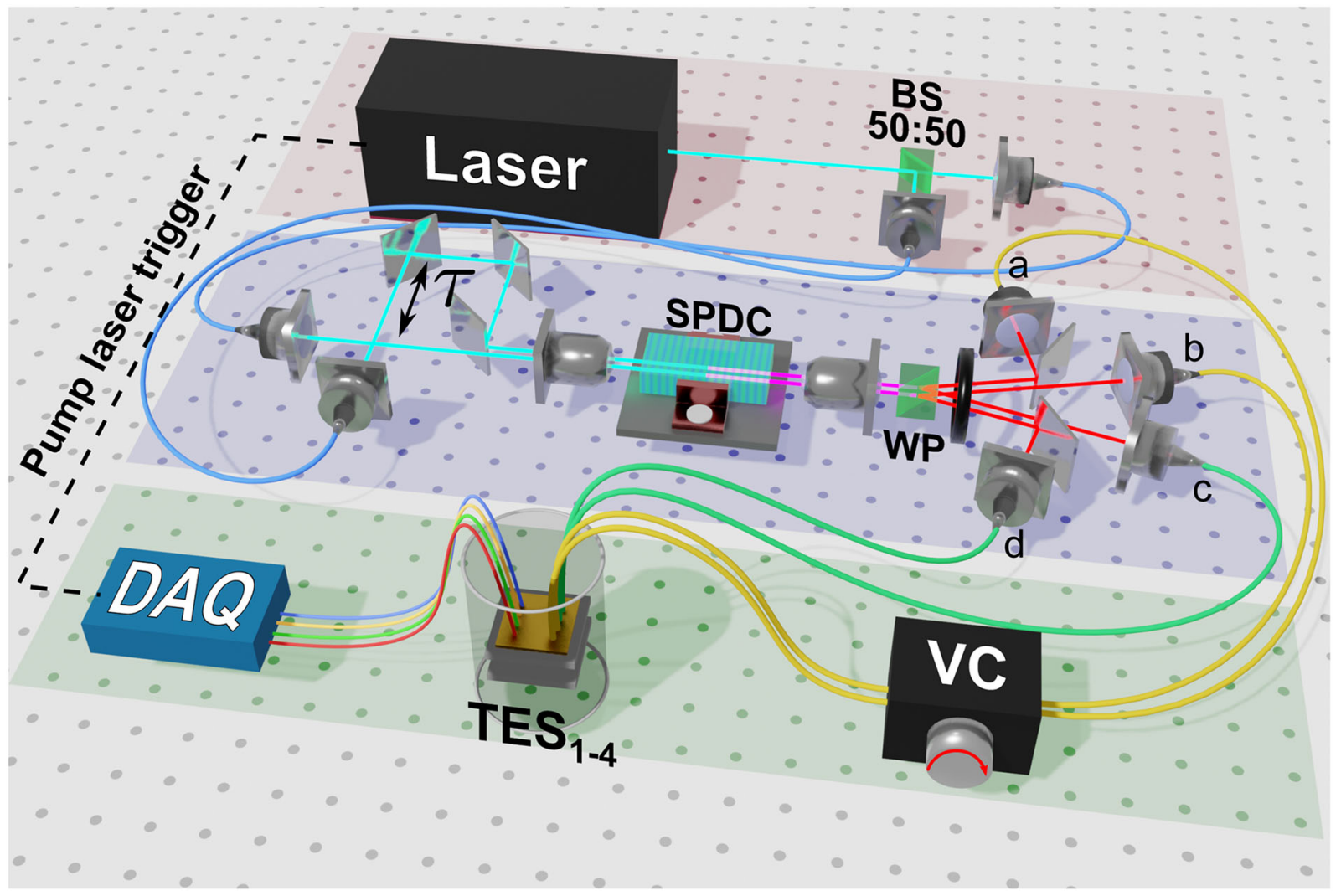

Fig. 3 Experimental integrated-photonics set-up for Fock state interference. Laser pulses (blue beams) centred at $775 \mathrm{~nm}$ pump two collinear type-Il phase-matched $8 \mathrm{~mm}$-long spontaneous parametric down-conversion (SPDC) waveguides written in a periodically poled KTP (PP-KTP) crystal. Each SPDC creates a two-mode photon-number correlated state (red beams). The modes are separated with a Wollaston prism (WP) into the modes $a-d$. They are filtered by bandpass filters tuned to the central wavelength $1554 \mathrm{~nm}$ for the signal modes $a$ and $b$, and $1546 \mathrm{~nm}$ for the idler modes $c$ and $d$. The idler beams are used for heralding the creation of the signal Fock states in $a$ and $b$ which interfere in a variable ratio phase-matched fibre coupler (VC). The VC allows us to set the ratio between 0 and 1 with an error of $\pm 1.5 \times 10^{-2}$. We used transition-edge sensors (TESs) with efficiency exceeding $90 \%$ for photon-number-resolved measurements in all modes ${ }^{33}$. The optimal temporal overlap at the VC is achieved by adjusting an optical path delay $\tau$. The data is analysed with a data acquisition unit (DAQ).

$|2 n-1\rangle_{a}|S-(2 n-1)\rangle_{b}$, respectively. To evidence this, a further simulation with input $|0\rangle_{a}|S\rangle_{b}$ was performed, where $S$ is an odd number, modelling the perfect transfer of Majorana modes between the two ends of a $p$-wave chain of $N=\frac{S+1}{2}$ atomic sites that is depicted in Fig. 2c. This is half the number of sites as in the $X Y$ spin chain, reflecting the fact that each physical fermion comprises a pair of Majoranas. The simulated dynamics also apply to one-dimensional arrays of photonic cavities ${ }^{41}$ where the effective superconducting pairing and Majorana modes arise from Kerr-type non-linearities within a Bose-Hubbard model. See Supplementary Note 5 for more details.

\section{Non-uniform transverse-field Ising chain}

One can also simulate a transverse-field Ising model, $H_{\mathrm{K}}=\frac{1}{2} \sum_{n=1}^{N}\left(\mu_{n} \sigma_{n}^{z}+2 t_{n} \sigma_{n}^{x} \sigma_{n+1}^{x}\right)$, since this is related to the $\mathrm{p}$ wave superconducting chain by a Jordan-Wigner transformation. Due to the non-uniform field $\mu_{n}$ and spin couplings $t_{n}$, the system inherits the perfect mirror reflection from the beam-splitter dynamics and allows for perfect state transfer after an interaction time $\theta=\pi$. We thus highlight a quantum spin network that allows perfect transfer, similar to the previously discussed XY model, but which has not been considered by previous authors. For example, to simulate the transfer of an excited spin between ends of a chain, one should interfere the state $\frac{1}{\sqrt{2}}\left(|0\rangle_{a}|S\rangle_{b}+|1\rangle_{a}|S-1\rangle_{b}\right)$ on a balanced beam splitter. Detailed derivations are presented in Supplementary Note 5.

\section{Experimental study}

Figure 3 shows the experimental integrated-photonics schema used for Fock state quantum simulations. Two pulsed spontaneous parametric down-conversion sources (SPDC) each generated independent two-mode photon-number-entangled states $|\Psi\rangle=\sum_{n=0}^{\infty} \sqrt{\langle n\rangle^{n}}|n, n\rangle$ with an average photon number $2\langle n\rangle=0.4$. For the pump repetition rate of $75 \mathrm{kHz}$ this led to $\sim 0.46$ five-photon (12 four-photon) Fock states created per minute in each arm of the SPDC, of which about 0.2 (6) reached the detectors due to ca. $50 \%$ losses in the set-up. One mode from each $|\Psi\rangle$ (the idlers, $c$ and $d$ ) was sent to a TES. Due to photonnumber entanglement in $|\Psi\rangle$ states, the outcomes of TESs, $I$ and $S-I$, heralded the creation of Fock states $|I\rangle_{a}$ and $|S-I\rangle_{b}$ in the signal modes $a$ and $b$.

We characterised the set-up to confirm the high degree of indistinguishability of these Fock states, the key issue for multiphoton HOM effect. We measured the standard HOM interference dip between both sources for a small mean photon number of the order of $10^{-4}$, and achieved the visibility $V_{\mathrm{HOM}}=$ $85.9 \%$. Next, we took a measurement of the second-order correlation function for each SPDC source separately and observed $g^{(2)} \geq 1.86 \approx 1+V_{\text {HOM }}$, which corroborates the previous result. An effective Schmidt mode number of $K=\frac{1}{g^{(2)}-1}=1.16$ proves our SPDC sources nearly single-mode.

The measured simulations are presented in Fig. 4. The data shown in Fig. $4 a$, b consists of $\sim 1.6 \times 10^{3}$ registered events, for each value of $r$, in which the total number of photons was $S=4$. The data in Fig. $4 c$ comprises $2.3 \times 10^{2}$ measurements, for each 

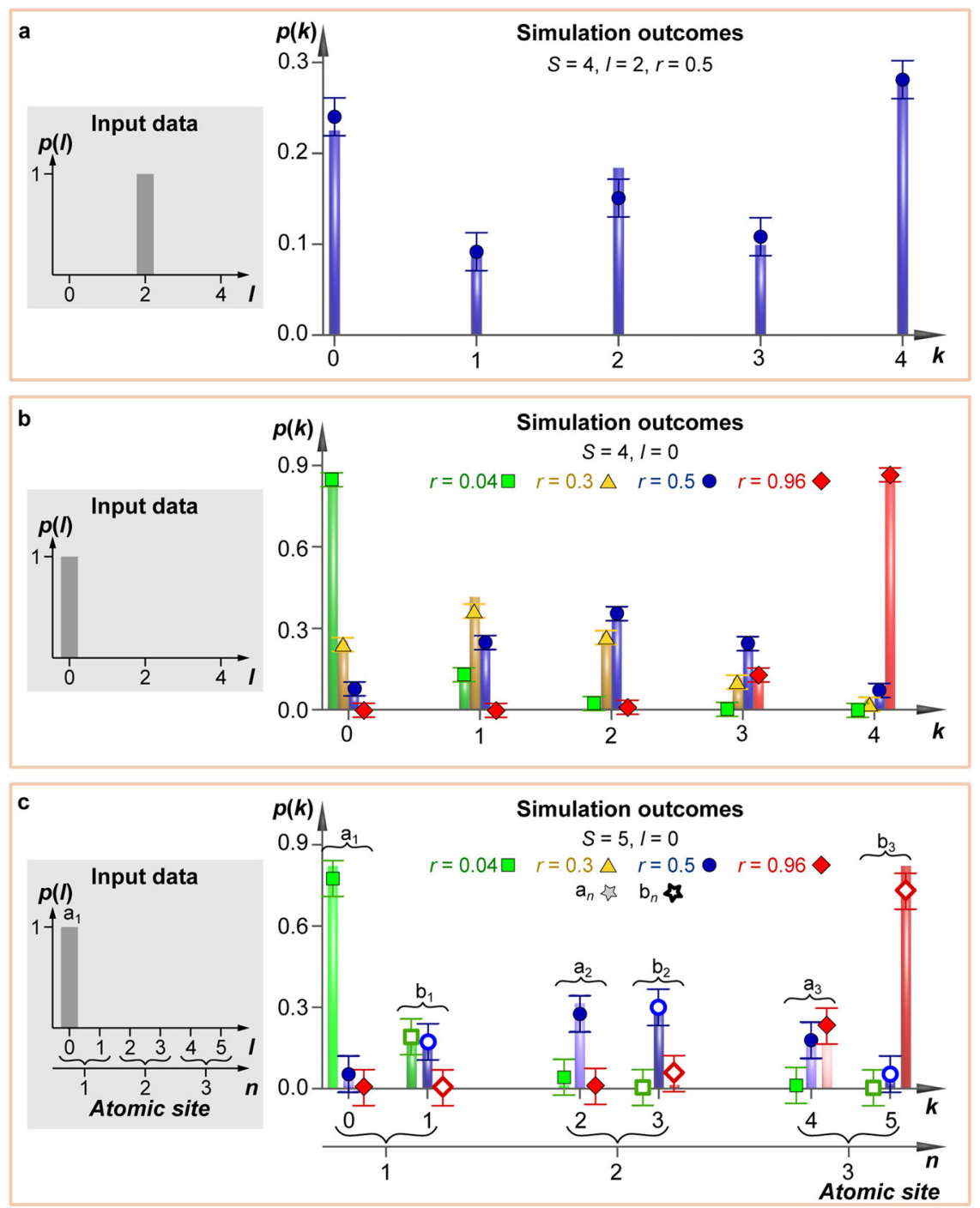

Fig. 4 Measured Fock state quantum simulations. The experimental simulation outcomes (symbols with error bars) are the directly measured photon statistics $p(k)$ resulting from interference of Fock states, $|I\rangle_{a}$ and $|S-I\rangle_{b}$, on a beam splitter of reflectivity $r$. The grey insets display the input data $l$, while the bars expected theoretical values of $p(k)$ obtained numerically. a Two two-photon Fock states $(S=4, I=2)$ interfering on a balanced beam splitter $(r=0.5)$ reveal an edge-state structure in the corresponding spin chain. $\mathbf{b}$ Interference of the vacuum and a four-photon number state $(S=4, I=0)$ observed for several gradually increasing reflectivities $r=0.04$ (green squares), 0.3 (orange triangles), 0.5 (blue circles) and 0.96 (red diamonds) models perfect wave packet transfer in the Kravchuk chain. c Interference of the vacuum and a five-photon number state $(S=5, I=0)$ observed for the same set of reflectivities models the transfer of Majorana fermions $a_{n}$ and $b_{n}$ across a p-wave superconducting chain of 3 sites. Errors were estimated as a square root inverse of the number of measurements.

value of $r$, in which $S=5$. We compared them with a numerical model based on Eq. (2) supplemented with the analysis of experimental imperfections, and found that they are in good agreement. Errors were estimated as a square root inverse of the number of measurements. See 'Methods' for details.

In Fig. 4a we show the photon statistics recorded by $\mathrm{TES}_{2-3}$ for the coupler splitting ratio $r=0.5$, conditioned on the heralded photon numbers $I=2$ and $S-I=2$ in modes $c$ and $d$. They directly model a zero-energy eigenmode of a non-linear SSH model described by $\left[\mathbf{H}_{\mathrm{BS}}\right]_{\mathrm{nm}}^{\text {Fock }}$, with emerging two weakly localised edge states. Figure $4 \mathrm{~b}$ depicts the statistics gathered for $I=0$ and $S-I=4$ for several splitting ratios: $r=0.04$ (green squares), 0.3 (orange triangles), 0.5 (blue circles) and 0.96 (red diamonds). It visualises perfect state transfer of the first spin excitation in the chain of 5 particles by means of continuous-time mirror reflection w.r.t. the chain centre. Figure $4 \mathrm{c}$ shows an experimental simulation of the perfect transfer of a Majorana fermion in a p-wave superconducting chain of 3 sites that is based on the statistics gathered for $I=0$ and $S-I=5$ for all the listed values of $r$.

\section{DISCUSSION}

Multi-particle Fock state interference is a compelling method in the field of quantum simulations, promising for studying noncrystalline topological materials, beyond the recently challenged bulk-edge correspondence theorem ${ }^{42,43}$. It allowed us to simulate systems as diverse as an XY spin chain and a non-linear SSH model, as well as the perfect transfer of Majorana fermions over a quantum wire, in a system that is not tied to a single-excitation subspace. The presented examples apply to a variety of systems such as superconducting nanowires ${ }^{44}$, disordered graphene quasi-1D nanoribbons ${ }^{45}$ and disordered cold atoms ${ }^{46}$. These may find applications in next-generation electronics ${ }^{47}$ and spintronics ${ }^{48}$ operating with almost no energy dissipation and speeds exceeding $100 \mathrm{GHz}$. 
Remarkably, photon-number-resolved detection we use introduces an effective non-linearity into our system, a feature which can be harnessed in simulated models. This effect is a result of combining quantum interference with a projective measurement, realized with TES detectors in our demonstration. It was recognized as an important component enabling universal quantum computation with linear optics ${ }^{28,29}$.

Multiphoton Fock states have been utilised in quantum simulations in a very limited capacity until now. The main focus has been on the successful manipulation of large numbers of single or two-photon states in bulk optics ${ }^{49-51}$, as well as in integrated platforms ${ }^{52-54}$. For example, output states of quantum walks in coupled waveguides mostly consist of single photons, with only a small fraction of two-photon states ${ }^{13-15,18}$. The perfect state transfer in an XY spin chain that we consider has also been simulated using both continuous ${ }^{16}$ and discrete-time ${ }^{17}$ singlephoton quantum walks. An advantage of this approach lies in easily engineered waveguide layouts which can be used to tune the couplings between spins ${ }^{18}$. However, a simulation of $N$ spins using single photons requires at least $N$ coupled waveguides, with the circuit length specifying the simulation duration. Thus, large waveguide arrays are needed, and it is difficult to vary the spinchain length or interaction time without fabricating many devices. Ensuring a high degree of indistinguishability of single photons coming from different sources also remains a challenge ${ }^{7}$.

In contrast, our simulations are done exclusively in Fock space, with Fock states of high photon number encoding all the information from input to output. Our method minimises the spatial complexity, needing only one interaction between two modes, irrespective of the size of the spin chain, while the simulation time may be easily tuned by the beam-splitter reflectivity. On the other hand, simulation of large chains requires the generation of high-order Fock states which is challenging due to losses, the stochastic nature of the heralding, and the existence of multiple modes in the SPDC process ${ }^{55}$. Experimental imperfections lead to a trade-off between the probability of Fock state generation and their fidelity. These errors are nevertheless minimized by engineering of robust PP-KTP waveguide sources and applying spatial and narrow spectral filtering, resulting in near-single-mode heralded Fock states. They are verified in the experiment by measuring the Glauber second-order correlation function $g^{(2)}$ and computing the Schmidt mode number $K$, which also takes possible phase errors into account ${ }^{56}$. Although currently the experimental generation of five-photon Fock states is already beyond the state of the art, it is soon expected to reach the level of tens of photons ${ }^{57}$. Recent demonstration of a photonic integrated circuit with a built-in four-mode interferometer, $8 \mathrm{~dB}$ total loss, and photon-number-resolving detection at all outputs, definitely sets high expectations for higher-dimensional Fock-state-based photonic computation in the near future ${ }^{58}$.

Qudit data encoding employed in our scheme brings yet another advantage. While in classical digital computation small errors in successive gates are easily corrected by rounding or truncating the signal to one of the allowed values (restandardisation), in the case of quantum circuits this is not possible and the errors accumulate 22 . Quantum error correction solutions, which address this problem, are difficult to realize in the current era of the Noisy Intermediate-Scale Quantum (NISQ) circuits ${ }^{23}$. The accumulated errors lead to drop of fidelity with the number of computation steps, which has been recently shown both theoretically $^{24,59,60}$ and in experiment ${ }^{25,27}$, including photonic setups $^{61}$. It is also foreseen that larger systems will suffer from higher individual gate errors due to technological constraints in controlling a large number of qubits ${ }^{26}$. In these circumstances, simplification introduced by the qudit encoding and a single quantum gate required for computation may lead to improvement of the precision. However, this comes at the cost of preparing a higher-order multiphoton state, and since higher photon Fock states are generated with lower fidelity ${ }^{55}$ this introduces a further source of error accumulation.

We emphasise that our approach can be generalised to more complex interferometric networks (e.g. combinations of beam tritters, quarters etc.) which allow one to simulate a wider class of Hamiltonians with more tunable parameters without changing the fundamental concepts ${ }^{62}$. Although it is an open intriguing question what additional systems may be simulated in this way, multiport interferometers such as beam tritters and quarters have already proven a useful tool for quantum simulations in the singlephoton regime ${ }^{63-65}$. In principle, multiport networks can implement any unitary evolution described by an $N \times N$ matrix ${ }^{66}$, a feature which has been very recently harnessed in photonic quantum computing chips ${ }^{58}$. Our approach can also be extended to higher dimensions by including additional degrees of freedom such as photon frequency and polarisation (see Supplementary Note 6). The scope of simulations could be further broadened by using input state superpositions $\sum_{l=0}^{S} x_{l}|I, S-I\rangle$ and altering the spin-chain couplings. Although preparation of such general superpositions poses a challenge in photonics, input states in the form of generalised Holland-Burnett states were experimentally obtained by interfering Fock states on a beam splitter ${ }^{67}$. Some other examples could be reached by heralding and conditional state preparation using more intricate interferometers. Merging our approach with coupled-waveguide set-ups is yet an unexplored and intriguing territory.

It would also be very interesting to implement our technique with quantum simulation platforms that are universal. For example, Fock states are also available in motional states of trapped ions up to 10 excitations ${ }^{68}$ and in the form of plaquette Fock states of atoms in optical lattices up to 4 excitations ${ }^{21}$. The range of accessible parameters controlling these systems could provide access to other complementary simulation models. Moreover, deterministic creation of an arbitrary superposition of Fock states has been demonstrated for trapped ions and superconducting resonators ${ }^{69}$. This would further expand the assortment of input states that could be used for simulation and may give birth to new fascinating results.

\section{METHODS}

\section{Characterisation of the set-up}

Each integrated SPDC source produced a two-mode weakly squeezed vacuum state $|\Psi\rangle=\sum_{n=0}^{\infty} \lambda_{n}|n, n\rangle_{s, i}$, where $s$ and $i$ denote two output modes, named the signal and idler, $\lambda_{n}=\frac{\tanh ^{n} g}{\cosh ^{2} g},\left|\lambda_{n}\right|^{2}$ is a probability of creation of a pair of $n$ photons and $g$ is the parametric gain. The average photon number in each mode of $|\Psi\rangle$ is $\langle n\rangle=\sinh ^{2} g$. The observed average photon number of $\langle n\rangle \approx 0.2$ amounts to $g=0.44$, which was sufficient to ensure the emission of multiphoton pairs. In this regime, one can approximate $\cosh g \approx 1$ and thus, $\lambda_{n} \approx \sinh ^{n} g=\sqrt{\langle n\rangle^{n}}$.

The TESs were operated at $70 \mathrm{mK}$, which allowed photon-number resolved measurements in all modes ${ }^{33}$.

The transmission losses in the set-up were estimated by means of Klyshko efficiency measurements. To this end, we set the reflectivity of variable coupler at $r=0.5$, and pumped each of the two SPDC sources separately at successively lower power values. The registered four-mode photon statistics were then binned into 'photon(s)/no-photon' datasets to mimic the use of standard binary detectors, e.g. avalanche photo-diodes, and we concluded the total efficiencies of the heralding modes $c$ and $d$ to be $\eta_{c}=50.3 \%$ and $\eta_{d}=48.5 \%$, respectively. The variable-coupler modes $a$ and $b$ exhibited a total efficiency of $\eta_{a}=21.6 \%$ and $\eta_{b}=20.6 \%$, respectively. These values result from the fact that each mode carried a $3 \mathrm{~dB}$ loss from the coupler itself and another $1 \mathrm{~dB}$ due to coupler insertion and fibre-to-fibre coupling losses. We estimated the transmission losses to be approximately of $50 \% \approx 3 \mathrm{~dB}$. Here $1 \mathrm{~dB}$ stands for the initial fibre incoupling loss due to spatial mode mismatch, while $0.25 \mathrm{~dB}$ stems from detectors inefficiencies, and the remaining loss is from three FC/PC fibreto-fibre couplers per mode as well as bending losses in the transmission fibres between the set-up and the detectors. 
The HOM visibility is computed using the formula $v^{(2)}=\frac{n_{\max }-n_{\min }}{n_{\max } n_{\text {min }}}$, where $n_{\max }$ and $n_{\min }$ are the maximal and minimal number of events registered by the TES detectors for the given photon number $S$. In the experiment for input $|2,2\rangle$ and $r=0.5$, we obtained $v^{(2)}=50.6 \% \pm 1.2 \%$, whereas for input $|0,4\rangle(|0,5\rangle)$ they were $99.1 \% \pm 2.5 \%(97.8 \% \pm 6.2 \%)$ for $r=0.04,87.6 \% \pm$ $2.2 \%(96.7 \% \pm 7.2 \%)$ for $r=0.3,65.7 \% \pm 1.7 \%(71.4 \% \pm 4.6 \%)$ for $r=0.5$ and $99.9 \% \pm 0.8 \%(98.6 \% \pm 7.2 \%)$ for $r=0.96$

\section{Error estimation}

In the experiment, each measurement results in a 4-tuple consisting of the number of photons registered by $\mathrm{TES}_{1-4}$, corresponding to photonnumber states in modes $a-d$ (Fig. 3). The tuple counts are stored in a database. The probability of detecting $k$ and $S-k$ photons in modes $a$ and $b$ is computed as $p(k)=N_{k} / N$, where $N_{k}$ is the database value retrieved for the key $(k, S-k, l, S-l)$ and $N$ is the total count of events characterised by the given total number of photons $S$. The measurement errors for each mode were estimated to $\Delta p=1 / \sqrt{N}$.

\section{Numerical model of experimental outcomes}

To assess the experimental results we developed a theoretical model which extended Eq. (2) by taking into account the influence of losses, multi-modeness of beams as well as inefficient photodetection.

Decoherence resulting from the first two effects was modelled by replacing the mode with a $b^{\dagger}$ superposition of the same mode $b^{\dagger}$ and an orthogonal one $b_{\perp}^{\dagger}$, i.e. $b^{\dagger} \rightarrow \cos y b^{\dagger}+\sin y b_{\perp}^{\dagger}$, where the parameter $y \in$ $\left(0, \frac{\pi}{2}\right)$ introduced weights and 'tuned' the distinguishability. This transformation led to the interference of $|I\rangle_{a}$ with a two-mode Fock state superposition $\sum_{n=0}^{S-I}\left(\begin{array}{c}S-I \\ n\end{array}\right)^{-1 / 2} \cos ^{n} y(\sin y)^{S-I-n}|n\rangle_{b}|S-I-n\rangle_{b \perp}$ instead of the single-mode Fock state $|S-I\rangle_{b}$, as before. Thus, effectively, some of the multiphoton states interfered with the vacuum state and this implemented the usual model describing particle loss. In our computations, we took $y=\arcsin \sqrt{(K-1) / K}$, where $K$ denoted the effective Schmidt mode number measured during the set-up characterisation. For $K=1.16$, we used $y=0.38$.

Realistic model of photodetection requires taking into account a probability of detecting $n_{d}$ photons when a Fock state $\left|n_{\text {in }}\right\rangle$ reaches a TES. It is given by $p_{\text {TES }}\left(n_{\text {in }}, n_{d}\right)=\left(\begin{array}{c}n_{\text {in }} \\ n_{d}\end{array}\right)(1-\eta)^{n_{\text {in }}-n_{d}} \eta^{n_{d}}$ where $n_{d} \leq n_{\text {in }}$ and $\eta$ is the detector efficiency. In our computations we first used a starting value of $\eta=0.7$ and then numerically optimised efficiencies for individual TESs to compensate for the uneven photon number distribution $p(k)$ seen in Fig. 3a. The programmes were written in Python using mpmath library.

\section{DATA AVAILABILITY}

All data needed to evaluate the conclusions in the paper are present in the paper and/or the Supplementary Information. Additional data available from authors upon request.

Received: 16 January 2021; Accepted: 30 April 2021;

Published online: 03 June 2021

\section{REFERENCES}

1. Song, B. et al. Observation of nodal-line semimetal with ultracold fermions in an optical lattice. Nat. Phys. 15, 911-916 (2019)

2. Harris, N. C. et al. Quantum transport simulations in a programmable nanophotonic processor. Nat. Photon. 11, 447-453 (2017)

3. Jordan, S. P., Lee, K. S. \& Preskill, J. Quantum algorithms for quantum field theories. Science 336, 1130-1133 (2012).

4. Khang, N. H. D., Ueda, Y. \& Hai, P. N. A conductive topological insulator with large spin Hall effect for ultralow power spin-orbit torque switching. Nat. Mater. 17, 808-813 (2018).

5. Šmejkal, L., Mokrousov, Y., Yan, B. \& MacDonald, A. H. Topological antiferromagnetic spintronics. Nat. Phys. 14, 242-251 (2018).

6. Nayak, C., Simon, S. H., Stern, A., Freedman, M. \& Das Sarma, S. Non-Abelian anyons and topological quantum computation. Rev. Mod. Phys. 80, 1083-1159 (2008).
7. Slussarenko, S. \& Pryde, G. J. Photonic quantum information processing: a concise review. Appl. Phys. Rev. 6, 041303 (2019).

8. Houck, A. A., Türeci, H. E. \& Koch, J. On-chip quantum simulation with superconducting circuits. Nat. Phys. 8, 292-299 (2012).

9. Kim, K. et al. Quantum simulation of the transverse Ising model with trapped ions. New J. Phys. 13, 105003 (2011).

10. Blatt, R. \& Roos, C. F. Quantum simulations with trapped ions. Nat. Phys. 8, 277-284 (2012).

11. Schreiber, A. et al. A $2 D$ quantum walk simulation of two-particle dynamics. Science 336, 55-58 (2012).

12. Fischer, U. R. \& Schützhold, R. Quantum simulation of cosmic inflation in twocomponent Bose-Einstein condensates. Phys. Rev. A 70, 063615 (2004).

13. Peruzzo, A. et al. Quantum walks of correlated photons. Science 329, 1500-1503 (2010).

14. Aspuru-Guzik, A. \& Walther, P. Photonic quantum simulators. Nat. Phys. 8 285-291 (2012)

15. Poulios, K. et al. Quantum walks of correlated photon pairs in two-dimensional waveguide arrays. Phys. Rev. Lett. 112, 143604 (2013).

16. Perez-Leija, A. et al. Coherent quantum transport in photonic lattices. Phys. Rev. $A$ 87, 012309 (2013).

17. Pitsios, I. et al. Photonic simulation of entanglement growth and engineering after a spin chain quench. Nat. Commun. 8, 1569 (2017).

18. Gräfe, M. \& Szameit, A. Integrated photonic quantum walks. J. Phys. B: At. Mol. Opt. Phys. 53, 073001 (2020).

19. Bloch, I., Dalibard, J. \& Nascimbène, S. Quantum simulations with ultracold quantum gases. Nat. Phys. 8, 267-276 (2012).

20. Flamini, F., Spagnolo, N. \& Sciarrino, F. Photonic quantum information processing a review. Rep. Prog. Phys. 82, 016001 (2019).

21. Islam, R. et al. Measuring entanglement entropy in a quantum many-body system. Nature 528, 77-83 (2015).

22. Landauer, R. Is quantum mechanics useful? Philos. Trans. R. Soc. A 353, 367-376 (1995).

23. Preskill, J. Quantum computing in the NISQ era and beyond. Quantum 2, 79 (2018).

24. Ma, L. \& Sanders, J. Markov chains for error accumulation in quantum circuits. Preprint at https://arxiv.org/abs/1909.04432 (2019).

25. $\mathrm{Ma}$, Y. et al. Error-transparent operations on a logical qubit protected by quantum error correction. Nat. Phys. 16, 827-831 (2020).

26. Fellous-Asiani, M., Chai, J. H., Whitney, R. S., Auffèves, A., \& Ng, H. K. Limitations in quantum computing from resource constraints. Preprint at https://arxiv.org/abs/ 2007.01966 (2020).

27. Reinhold, P. et al. Error-corrected gates on an encoded qubit. Nat. Phys. 16 822-826 (2020).

28. Knill, E., Laflamme, R. \& Milburn, G. J. A scheme for efficient quantum computation with linear optics. Nature 409, 46-52 (2001).

29. Scheel, S., Nemoto, K., Munro, W. J. \& Knight, P. L. Measurement-induced nonlinearity in linear optics. Phys. Rev. A 68, 032310 (2003).

30. Su, W. P., Schrieffer, J. R. \& Heeger, A. J. Solitons in polyacetylene. Phys. Rev. Lett. 42, 1698-1701 (1979).

31. Yao, N. Y. et al. Quantum logic between remote quantum registers. Phys. Rev. A 87, 022306 (2013).

32. Kitaev, A. Y. Unpaired Majorana fermions in quantum wires. Phys.-Usp. 44, 131 (2001).

33. Gerrits, T. et al. On-chip, photon-number-resolving, telecommunication-band detectors for scalable photonic information processing. Phys. Rev. A 84, 060301 (2011).

34. Hong, C. K., Ou, Z. Y. \& Mandel, L. Measurement of subpicosecond time intervals between two photons by interference. Phys. Rev. Lett. 59, 2044-2046 (1987).

35. Campos, R. A., Saleh, B. E. A. \& Teich, M. C. Quantum-mechanical lossless beam splitter: SU(2) symmetry and photon statistics. Phys. Rev. A 40, 1371-1384 (1989).

36. Stobińska, M. et al. Quantum interference enables constant-time quantum information processing. Sci. Adv. 5, eaau9674 (2019).

37. Atakishiyev, N. M. \& Wolf, K. B. Fractional Fourier-Kravchuk transform. J. Opt. Soc Am. A 14, 1467-1477 (1997).

38. Gorlach, M. A. \& Slobozhanyuk, A. P. Nonlinear topological states in the Su-Schrieffer-Heeger model. Nanosystems: Phys. Chem. Math. 8, 695-700 (2017).

39. Christandl, M., Datta, N., Ekert, A. \& Landahl, A. J. Perfect state transfer in quantum spin networks. Phys. Rev. Lett. 92, 187902 (2004).

40. Christandl, M. et al. Perfect transfer of arbitrary states in quantum spin networks. Phys. Rev. A 71, 032312 (2005).

41. Bardyn, C.-E. \& İmamoğlu, A. Majorana-like modes of light in a one-dimensional array of nonlinear cavities. Phys. Rev. Lett. 109, 253606 (2012).

42. Downing, C. A., Sturges, T. J., Weick, G., Stobińska, M. \& Moreno, L. M. Topological phases of polaritons in a cavity waveguide. Phys. Rev. Lett. 123, 217401 (2019). 
43. Pocock, S. R., Huidobro, P. A. \& Giannini, V. Bulk-edge correspondence and long-range hopping in the topological plasmonic chain. Nanophotonics 8, 1337-1347 (2019).

44. Diez, M., Dahlhaus, J. P., Wimmer, M. \& Beenakker, C. W. J. Andreev reflection from a topological superconductor with chiral symmetry. Phys. Rev. B 86, 094501 (2012).

45. Han, M. Y., Özyilmaz, B., Zhang, Y. \& Kim, P. Energy band-gap engineering of graphene nanoribbons. Phys. Rev. Lett. 98, 206805 (2007).

46. Pinheiro, F. \& Larson, J. Disordered cold atoms in different symmetry classes. Phys. Rev. A 92, 023612 (2015).

47. McCaughan, A. N. \& Berggren, K. K. A superconducting-nanowire three-terminal electrothermal device. Nano Lett. 14, 5748-5753 (2014).

48. Nautiyal, T., Rho, T. H. \& Kim, K. S. Nanowires for spintronics: a study of transitionmetal elements of groups 8-10. Phys. Rev. B 69, 193404 (2004).

49. Huang, Y.-F. et al. Experimental generation of an eight-photon Greenberger-Horne-Zeilinger state. Nat. Commun. 2, 546 (2011).

50. Wang, X.-L. et al. Experimental ten-photon entanglement. Phys. Rev. Lett. 117, 210502 (2016).

51. Zhong, H. S. et al. 12-photon entanglement and scalable scattershot Boson sampling with optimal entangled-photon pairs from parametric downconversion. Phys. Rev. Lett. 121, 250505 (2018).

52. Caspani, L. et al. Integrated sources of photon quantum states based on nonlinear optics. Light Sci. Appl. 6, e17100 (2017).

53. Blanco-Redondo, A., Bell, B., Oren, D., Eggleton, B. J. \& Segev, M. Topological protection of biphoton states. Science 362, 568-571 (2018).

54. Wang, $\mathrm{H}$. et al. Boson sampling with 20 input photons and a 60 -mode interferometer in a $10^{14}$-dimensional Hilbert space. Phys. Rev. Lett. 123, 250503 (2019).

55. Tiedau, J. et al. Scalability of parametric down-conversion for generating higherorder Fock states. Phys. Rev. A 100, 041802 (2019).

56. Eckstein, A., Christ, A., Mosley, P. J. \& Silberhorn, C. Highly efficient single-pass source of pulsed single-mode twin beams of light. Phys. Rev. Lett. 106, 013603 (2011).

57. Harder, G. et al. Single-mode parametric-down-conversion states with 50 photons as a source for mesoscopic quantum optics. Phys. Rev. Lett. 116, 143601 (2016).

58. Arrazola, J. M. et al. Quantum circuits with many photons on a programmable nanophotonic chip. Nature 591, 54-60 (2021).

59. Gulliksen, J., Dasari, D. B. R. \& Mølmer, K. Characterization of how dissipation and dephasing errors accumulate in quantum computers. EPJ Quantum Technol. 2, 4 (2015).

60. Reiner, J.-M. et al. Effects of gate errors in digital quantum simulations of fermionic systems. Quantum Sci. Technol. 3, 045008 (2018).

61. Boutari, J. et al. Large scale quantum walks by means of optical fiber cavities. J. Opt. 18, 094007 (2016).

62. Osawa, S., Simon, D. S. \& Sergienko, A. V. Directionally-unbiased unitary optical devices in discrete-time quantum walks. Entropy 21, 853 (2019).

63. Spagnolo, N. et al. Three-photon bosonic coalescence in an integrated tritter. Nat. Commun. 4, 1606 (2013).

64. Spagnolo, N. et al. Quantum interferometry with three-dimensional geometry. Sci. Rep. 2, 862 (2012).

65. Menssen, A. J. et al. Distinguishability and many-particle interference. Phys. Rev. Lett. 118, 153603 (2017).

66. Reck, M., Zeilinger, A., Bernstein, H. J. \& Bertani, P. Experimental realization of any discrete unitary operator. Phys. Rev. Lett. 73, 58-61 (1994).

67. Thekkadath, G. S. et al. Quantum-enhanced interferometry with large heralded photon-number states. npj Quantum Inf. 6, 89 (2020).

68. Ding, S., Maslennikov, G., Hablützel, R. \& Matsukevich, D. Cross-Kerr nonlinearity for phonon counting. Phys. Rev. Lett. 119, 193602 (2017).
69. Hofheinz, M. et al. Synthesizing arbitrary quantum states in a superconducting resonator. Nature 459, 546-549 (2009).

\section{ACKNOWLEDGEMENTS}

T.S., T.M., A.B. and M.S. were supported by the Foundation for Polish Science 'First Team' project No. POIR.04.04.00-00-220E/16-00 (originally: FIRST TEAM/2016-2/17) and the National Science Centre 'Sonata Bis' project No. 2019/34/E/ST2/00273. A.E. and I.W. were supported by the Engineering and Physical Sciences Research Council project No. EP/K034480/1.

\section{AUTHOR CONTRIBUTIONS}

Both T.S. and T.M. contributed equally. T.S., T.M., A.B. and M.S. developed the theory while A.E., W.R.C., W.S.K. and I.A.W. were responsible for realisation of the experiment. J.J.R., S.W.N., T.G. and A.L. delivered and maintained the transition-edge sensor detection system. A.B., T.S. and T.M. developed the software and performed numerical computations. A.B. prepared the plots. All the co-authors wrote up the manuscript.

\section{COMPETING INTERESTS}

The authors declare no competing interests.

\section{ADDITIONAL INFORMATION}

Supplementary information The online version contains supplementary material available at https://doi.org/10.1038/s41534-021-00427-w.

Correspondence and requests for materials should be addressed to M.S.

Reprints and permission information is available at http://www.nature.com/ reprints

Publisher's note Springer Nature remains neutral with regard to jurisdictional claims in published maps and institutional affiliations.

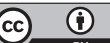

Open Access This article is licensed under a Creative Commons Attribution 4.0 International License, which permits use, sharing, adaptation, distribution and reproduction in any medium or format, as long as you give appropriate credit to the original author(s) and the source, provide a link to the Creative Commons license, and indicate if changes were made. The images or other third party material in this article are included in the article's Creative Commons license, unless indicated otherwise in a credit line to the material. If material is not included in the article's Creative Commons license and your intended use is not permitted by statutory regulation or exceeds the permitted use, you will need to obtain permission directly from the copyright holder. To view a copy of this license, visit http://creativecommons. org/licenses/by/4.0/.

(c) The Author(s) 2021 